\title{
Aplicabilidade e exequiibilidade da técnica de interlocking nail como tratamento de fraturas diafisárias de fêmur em cães
}

\section{Applicability and feasibility of the interlocking nail technique for treatment of diaphyseal femoral fractures in dogs}

\section{Aplicabilidad y ejecución de la técnica de clavo bloqueado como tratamiento de las fracturas diafisarias de fémur en perros}

\section{Alexandre Schmaedecke, ${ }^{1}$ Vanessa Couto de Magalhães Ferraz, ${ }^{2}$ Cássio Ricardo Auada Ferrigno $^{3}$}

Departamento de Cirurgia da Faculdade de Medicina Veterinária e Zootecnia da Universidade de São Paulo (FMVZ/USP). São Paulo, SP, Brasil

\section{Resumo}

Objetivo: Analisar a técnica de interlocking nail como tratamento de fraturas diafisárias de fêmur em cães, levando em consideração a comparação com técnicas consagradas de reparação óssea. Fontes Consultadas: Foram pesquisadas as bases de dados LILACS, DEDALUS, PUBMED, e o acervo da Biblioteca da Faculdade de Medicina Veterinária e Zootecnia da Universidade de São Paulo pelo período retrospectivo de seis anos. Síntese dos Dados: A técnica de interlocking nail tem sido proposta como método seguro, com menor tempo de recuperação e melhores resultados no tratamento de fraturas diafisárias de fêmur em cães por atuar sobre o vetor de força do osso, respeitando o mecanismo biológico de reparação de fraturas. Conclusões: A técnica de interlocking nail mostra-se aplicável e exeqüível no tratamento de fraturas diafisárias de fêmur em cães.

Palavras-chave: Consolidação da fratura. Fixação de fratura. Fixadores internos. Pinos ortopédicos. Fraturas do fêmur. Cães.

' Doutorando do Departamento de Cirurgia da FMVZ/USP. CRMV-PR 4439

Aluna de graduação da FMVZ/USP

${ }^{3}$ Professor Doutor do Departamento de Cirurgia da FMVZ/USP. CRMV-SP 6835 


\section{Introdução}

Fraturas de ossos longos são, sem dúvida, um dos principais pontos de tratamento ortopédico na Medicina Veterinária. Daquelas, as fraturas de fêmur são responsáveis por aproximadamente $20 \%$ a $25 \%$ do total de casos apresentados por cães e gatos. ${ }^{1}$ Harasen $^{1}$ (2003) apresenta estudo retrospectivo em que $45 \%$ do total de fraturas de ossos longos atendidas correspondiam ao fêmur (Figura 1), enquanto $26 \%$ eram fraturas tibiais. Essas fraturas geralmente ocorrem por acidentes automobilísticos, quedas ou acidentes com armas de fogo.

Muitas técnicas são descritas para o tratamento desses quadros, incluindo uso de pinos intramedulares, fios de cerclagem, fixadores externos e placas parafusadas, ou combinações destas (LARIN et al., ${ }^{2}$ 2001). Pinos intramedulares têm sido usados devido à sua praticidade e baixo custo em termos de estabilização de fraturas diafisárias de cães e gatos (PRESNEL, ${ }^{3}$ 1978). No entanto, são comuns as complicações cirúrgicas decorrentes da instabilidade do foco de fratura, migração do implante, rotação e encurtamento do osso afetado (DUHAUTOIS, ${ }^{4} 2003$; KHAN, $\left.{ }^{5} 1995\right)$.

O conceito de recuperação biológica das fraturas foi introduzido há alguns anos, no intuito de preservar, ao máximo possível, a integridade vascular dos fragmentos ósseos e, ao mesmo tempo, prover uma fixação capaz de manter o alinhamento e o comprimento ósseos durante o período de reparação (ARON et al., ${ }^{6}$ 1995; BERNARDÉ et al., ${ }^{7} 2002$; PERREN, ${ }^{8}$ 1991; WOO et al., ${ }^{9}$ 1984). Fraturas tratadas por este método são reduzidas e estabilizadas a céu fechado, utilizando, em Medicina Humana, apenas fluoroscopia e pequenas incisões para colocação dos implantes. O alinhamento dos fragmentos ósseos maiores e articulações predomina sobre a reconstrução anatômica. Em Medicina Veterinária, pelo pouco acesso aos exames fluoroscópicos, instituiu-se a técnica do "abra, mas não toque", que consiste em mínimas incisões, com o menor contato possível com os tecidos moles adjacentes ao foco de fratura, exclusivamente para observar o quadro apresentado e fixar o implante (McLAUGHLIN, ${ }^{10}$ 1999; PALMER, ${ }^{11} 1999$; HORSTMAN et al., ${ }^{12}$ 2004). Esta abordagem preserva o potencial osteogênico do hematoma provocado pela fratura (BERNARDÉ et al., ${ }^{7} 2001$ ), minimiza a perda de vascularização, reduz o tempo cirúrgico e diminui a possibilidade de infecção pósoperatória (HORSTMAN et al., ${ }^{12}$ 2004); McCLURE et al., ${ }^{13}$ 1998; LOPEZ et al., ${ }^{14}$ 2001).

A fixação intramedular como fixação interna de fraturas é conhecida e utilizada desde o século XVI, e a técnica de interlocking nail (IN) foi primeiramente descrita, em humanos, no princípio dos anos 50, por Modney (DUELAND et al., ${ }^{15}$ 1999; GUPTA, ${ }^{16}$ 2001). Haste bloqueada ou interlocking nail (IN) são pinos intramedulares com furos transversais posicionados em distâncias padronizadas, permitindo, através deles, realizar o bloqueio dos movimentos rotacionais e axiais, por meio de parafusos. O pino de IN é colocado de forma intramedular e os parafusos são fixados proximal e distalmente à fratura. Além de prevenir os movimentos responsáveis pelas complicações mencionadas para fixação com pinos intramedulares, o uso de IN é biomecanicamente vantajoso em relação às técnicas de imobilização por placa parafusada, por atuar ao longo do eixo mecânico central do osso (MOSES et al., ${ }^{17} 2002$ ), além de preservar os conceitos de padrões biológicos de osteossíntese (DUHAUTOIS, ${ }^{4}$ 2003).

Em pacientes humanos, a fixação por IN tem suplantado o uso de placas parafusadas em muitas fraturas diafisárias de fêmur, tíbia e úmero. Essa preferência justifica-se pela facilidade de aplicação, manutenção da vascularização dos fragmentos e segurança de técnica, uma vez que é aplicada com auxílio de fluoroscopia na colocação dos pinos transcorticais. Esse procedimento minimiza o acesso cirúrgico e torna efetivamente precisa a técnica, diminuindo também a morbidade pós-cirúrgica (MOSES et al., ${ }^{17}$ 2002). Suas indicações incluem fraturas diafisárias fechadas ou abertas (tipo I), simples ou cominutivas, pseudoartroses infectadas ou não, além de osteotomias corretivas de alongamento, encurtamento ou derrotacionais. 
Este sistema foi adaptado à necessidade da Medicina Veterinária, tendo sido criado, em 1989, modelo específico por Dueland ${ }^{15}$ (Innovative Animal Products $^{\circledR}$ ), comercializado desde 1994. O sistema veterinário utiliza um guia acoplado à extremidade proximal do pino intramedular para localização exata dos pontos de inserção dos parafusos, permitindo sua aplicação sem a utilização de fluoroscópios (McLAUGHLIN, ${ }^{10}$ 1999). Três gerações de pinos já foram desenvolvidas, e a última consiste em pinos de 6 ou $8 \mathrm{~mm}$ de diâmetro, com cinco diferentes comprimentos (140,160,185, 205 e $230 \mathrm{~mm})$, apresentando dois furos proximais e dois furos distais para bloqueio com parafusos de 2,7 ou 3,5 mm para animais de companhia. Estes furos estão localizados a uma distância fixa de 11 ou $22 \mathrm{~mm}$ entre si. $\mathrm{Na}$ porção proximal do pino existe fenda para alocação do extensor que pode ser de 80 ou $120 \mathrm{~mm}$ de comprimento. O mais curto é utilizado em tratamento de fraturas de úmero e fêmur, enquanto o mais longo é utilizado em fraturas de tíbia, para evitar qualquer contato do equipamento com os côndilos da tróclea do fêmur ou patela. O pino é inserido por via normógrada, sendo previamente escolhido pelo comprimento do osso visualizado em radiografia, por meio de modelo específico impresso em filme transparente. Este é utilizado como molde colocado por sobre o filme radiográfico. O extensor é então disposto e os parafusos são alocados de acordo com marcações específicas do guia que, por sua vez, está fixado ao pino pelo extensor (DUELAND et al., ${ }^{15}$ 1999).

Algumas descrições já são encontradas quanto ao uso desta técnica em animais. Dueland et al., ${ }^{15}$ em 1999, descreveram a aplicação de IN em tratamento de diferentes fraturas diafisárias em 134 cães, sendo 92 fraturas de fêmur, 23 de tíbia e 19 de úmero, 70 delas classificadas como instáveis. Como resultado, $83 \%$ dos quadros foram tratados sem nenhuma complicação, e em apenas um caso não houve remissão do quadro. Moses et al. ${ }^{17}$ descreveram, em 2002, o tratamento de 21 fraturas de úmero com utilização da técnica, com $86 \%$ de recuperação sem complicações. Langley-Hobbs e Friend, ${ }^{18}$ em 2002, descreveram o tratamento de fratura de fêmur em peru, com completa remissão do quadro e retorno da função do membro após duas semanas de confinamento.

O uso desta modalidade de técnica tem sido mais efetivo na reparação de fraturas diafisárias altamente cominutivas em relação às demais técnicas propostas. A neutralidade da colocação do pino, tanto quanto sua geometria, promove a rigidez necessária ao bloqueio das forças, diminuindo a fatiga provocada por elas sobre o material. A utilização de placas parafusadas em fraturas cominutivas causa aumento da fatiga no implante por sua alocação em posição excêntrica, tornando maiores as forças de encurvamento e deslocamento lateral. Além disso, os pinos de IN podem ser implantados por acessos cirúrgicos menores que as placas, minimizando a manipulação e a incisão de tecidos moles adjacentes, e implicando na manutenção da integridade biológica do foco da fratura.

Em relação ao uso de fixadores externos, os pinos de IN apresentam vantagens ainda maiores, pois não necessitam de manejo ambulatorial desse item com tanta freqüência. Se comparada ao uso de pino intramedular e cerclagem associados, a técnica de IN apresenta resultados consideravelmente melhores, uma vez que a perda da cerclagem por quebra do fio ocorre freqüentemente, além da pouca estabilidade rotacional promovida por aquela associação (DUELAND et al., ${ }^{15}$ 1999).

Saravanan et al., ${ }^{19,20}$ em 2002, desenvolveram estudo histopatológico da formação de calo ósseo após diferentes formas de fixação interna de fraturas altamente cominutivas em cães. Apresentaram como resultado a incapacidade de estabilização óssea inerente à utilização de pino intramedular associado a fios de cerclagem, enquanto resultados extremamente satisfatórios foram observados no grupo testado após implantação de hastes bloqueadas de IN.

Mesmo o tratamento de animais portadores de quadros ortopédicos mais complexos já foi descrito por intermédio da utilização de implantes de haste bloqueada. Em 2001, ElShafie et al. ${ }^{21}$ descreveram 
o uso desta modalidade em correção de fratura ipsiateral de diáfise e colo femoral em nove pacientes humanos, tendo apresentado bons resultados em oito, e união retardada em apenas um caso.

Embora a haste bloqueada revele resultados expressivamente melhores se comparada às demais técnicas já consagradas para o tratamento de fraturas cominutivas, algumas restrições foram relatadas. Gupta $^{16}$ (2001) indicou que, apesar de promover excelente bloqueio contra as forças de rotação, compressão e encurvamento, esta técnica impede micromovimentações do foco de fratura, o que, em alguns casos, funcionaria como estímulo fisiológico para o desenvolvimento do calo ósseo.

O objetivo do presente estudo é analisar a técnica de interlocking nail como tratamento de fraturas diafisárias de fêmur em cães, comparando-a com técnicas consagradas de reparação óssea, divulgadas na literatura dos últimos seis anos.

Atualmente, os kits de implantação já estão disponíveis no mercado brasileiro, permitindo o acesso dos clínicos a essa técnica, que vem para facilitar a prática ortopédica dentro de suas rotinas.

\section{Implantação da Haste Bloqueada $^{15}$}

O tamanho da haste será determinado anteriormente ao procedimento por exame radiográfico. A haste deve preencher toda a extensão do canal medular, devendo seu diâmetro ocupar a maior parte dele. Em relação à curvatura caudal do canal medular, no fêmur, a atenção deve ser redobrada enquanto se posiciona a haste na porção distal do osso citado, para que ele não seja forçado em direção à cortical. O osso deve ser previamente fresado, para que nenhuma força adicional seja necessária durante a introdução da haste.

Para inserção da haste, podem ser adotadas as vias normógrada ou retrógrada. A rotação da haste se dá até que os furos estejam alinhados segundo a intenção do cirurgião (geralmente a orientação médiolateral é escolhida). Neste momento, o guia é encaixado e fixado à haste por meio de extensor acoplado à sua borda cranial.

Em relação aos bloqueios, utilizam-se as marcações específicas do guia para o tamanho do pino escolhido. Com auxílio de manga específica, inserida no local adequado do guia, alinha-se a perfuratriz elétrica ao ponto exato de fixação transcortical. $\mathrm{O}$ furo é feito através das duas corticais, com a broca alcançando a cortical distal por transposição do orifício existente na haste. As duas corticais são então perfuradas, macheadas e o parafuso fixado, bloqueando a haste. Este procedimento é repetido para todos os bloqueios. O guia é, assim, desengatado e a síntese dos tecidos moles se dá pelos padrões consagrados em cirurgia (Figura 2).
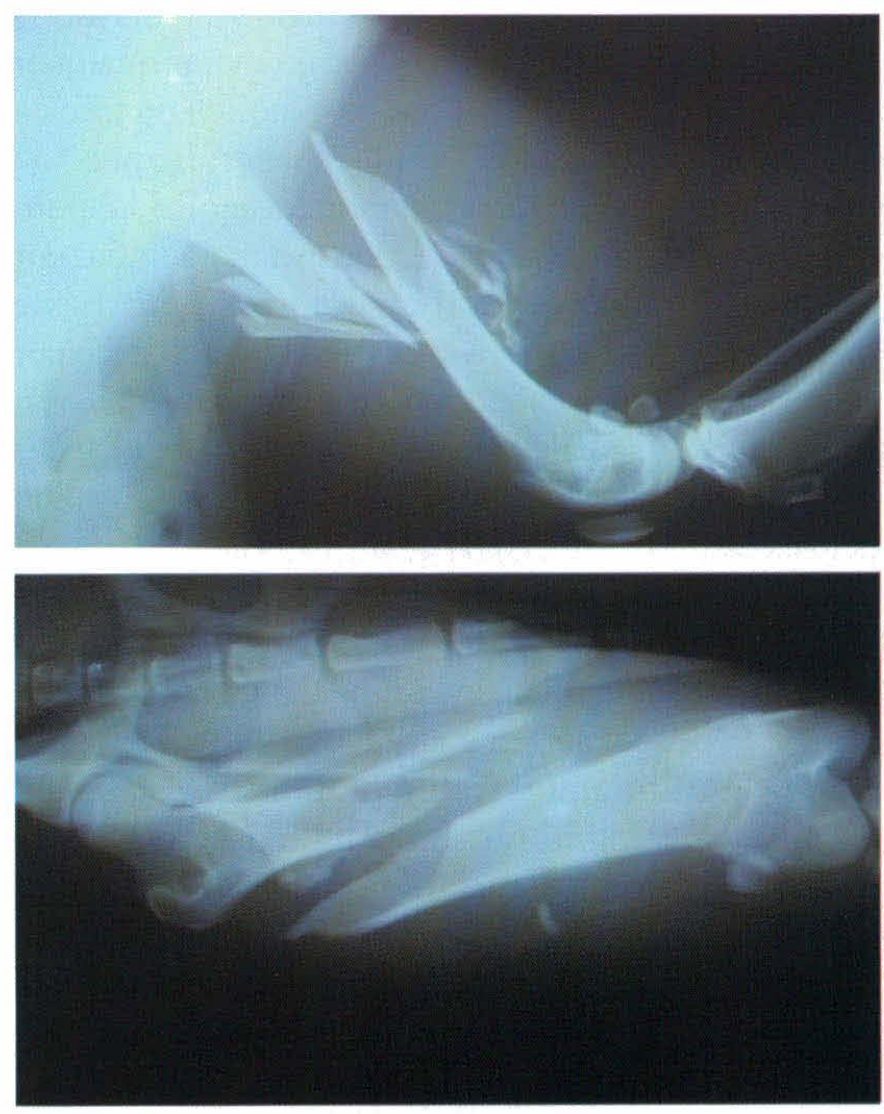

Figura 1 - Imagens radiográficas de fratura cominutiva de fêmur em cão da raça Pastor Alemão de $28 \mathrm{~kg}$, quatro anos de idade. (Setor de Cirurgia do Hospital Veterinário da FMVZ/USP) 


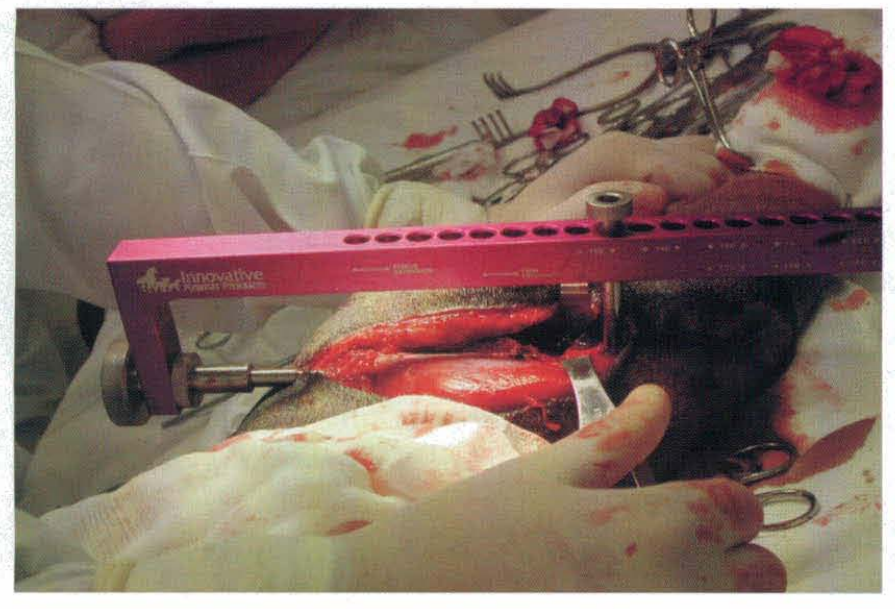

Figura 2 - Imagem fotográfica transcirúrgica evidenciando implantação da haste de interlocking nail. (Setor de Cirurgia do Hospital Veterinário da FMVZ/USP)

\section{Considerações Finais}

Falhas na reparação de fraturas são bastante evidentes pelo não-cumprimento das regras básicas de cirurgia ortopédica. Embora cada caso apresente um tratamento específico, que ainda assim pode ser alterado no momento da intervenção, é sabido, pelas regras do chamado "mecanismo biológico de reparação de fraturas", que quanto menor for a manipulação do sítio de fratura (e menor a exposição deste local) melhores serão os resultados obtidos. Portanto, a escolha de uma técnica que anule ou diminua ao máximo as forças de torção, tração e cisalhamento, atuantes sobre o foco de fratura, aumenta significativamente a taxa de sucesso do tratamento.

Deste modo, a utilização de hastes bloqueadas em fraturas diafisárias de fêmur em cães tem-se demonstrado extremamente aplicável, uma vez que, além de respeitar todos os mecanismos acima descritos, diminui significativamente o tempo cirúrgico e apresenta resultados efetivamente melhores se comparados às técnicas consagradas de reparação óssea, por atuar no bloqueio das forças citadas, exatamente sobre seu vetor, conferindo estabilidade superior até mesmo ao uso de placas parafusadas (Figura 3).
Embora os resultados demonstrem a alta eficácia do implante, o uso da haste bloqueada apresenta como restrições as fraturas localizadas nas extremidades dos ossos longos, impossibilitando sua fixação. Deste modo, a técnica de interlocking nail não tem a função de substituir nenhuma técnica consagrada, mas, sim, tornar-se uma opção acessível, prática e de resultados efetivos na rotina da ortopedia de pequenos animais.
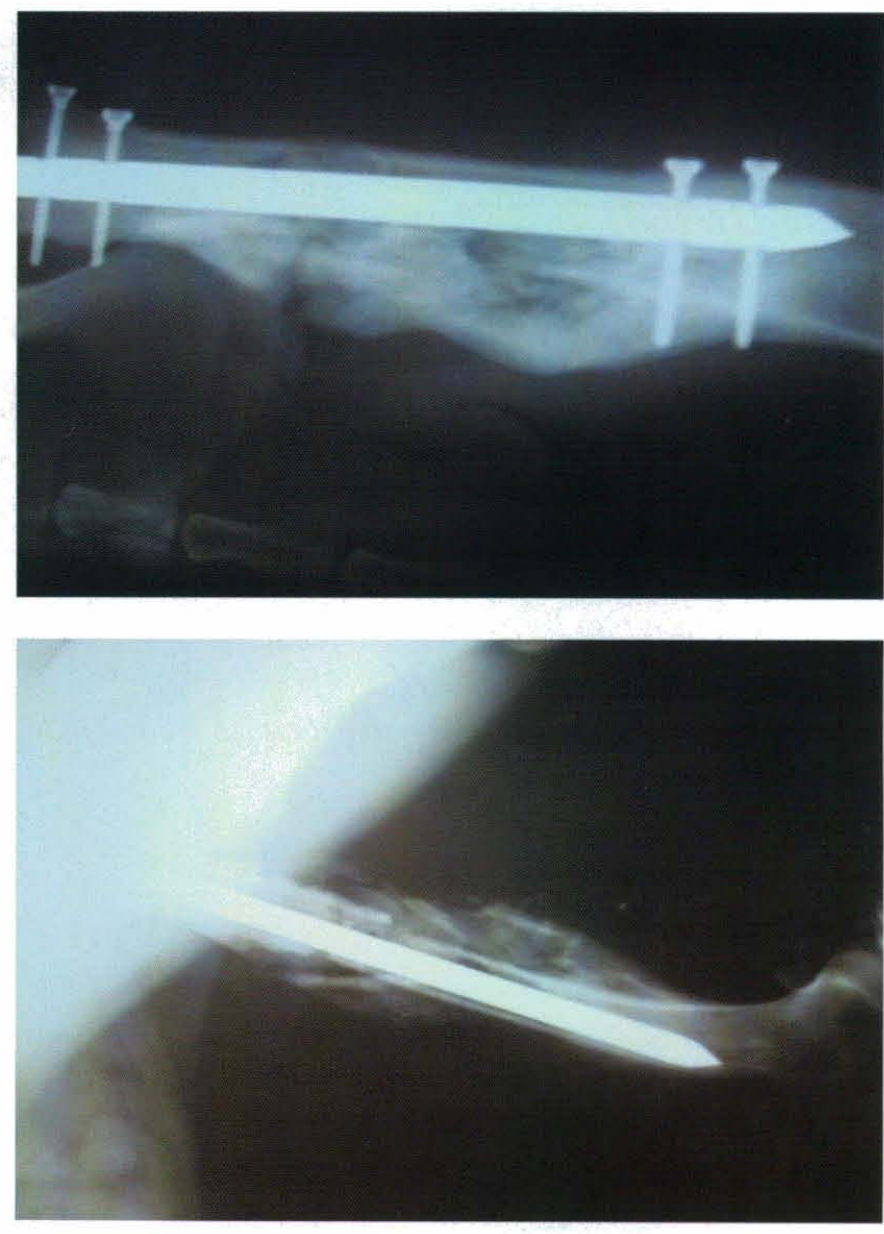

Figura 3 - Imagens radiográficas com 60 dias de pósoperatório, evidenciando expressiva formação de calo ósseo. (Setor de Cirurgia do Hospital Veterinário da FMVZ/USP)

\section{Agradecimentos} Brazil.

À empresa Innovative Veterinary Products, 


\section{Abstract}

Objective: To analyze the interlocking nail technique for treatment of diaphyseal femoral fractures in dogs, comparing it to consecrated techniques of bone repair. Data Sources: LILACS, DEDALUS, PUBMED databases and the collection of the library of the Faculdade de Medicina Veterinária e Zootecnia da Universidade de São Paulo (School of Veterinary Medicine and Animal Science of the University of São Paulo), São Paulo, SP, Brazil, were searched, covering a retrospective period of 6 years. Data Synthesis: The interlocking nail technique is currently being proposed as a safe method for treatment of diaphyseal femoral fractures in dogs, with shorter recovery time and better results, once it acts on the forces vector of the bone, respecting the biological mechanism of fracture healing. Conclusions: The interlocking nail technique is applicable and feasible for treatment of diaphyseal femoral fractures in dogs.

Keywords: Fracture healing. Fracture fixation. Internal fixators. Bone nails. Femoral fractures. Dogs.

\section{Resumen}

Objetivo: Analizar la técnica de clavo bloqueado para tratamiento de fracturas diafisarias de fémur en perros, comparando las técnicas consagradas del reparo óseo. Fuentes Consultadas: Se investigó en las bases de datos LILACS, DEDALUS, PUBMED y en el acervo de la Biblioteca de la Facultad de Medicina Veterinaria y Zootecnia de la Universidad de São Paulo, São Paulo, SP, Brasil, durante el periodo retrospectivo de 6 años. Síntesis de los Datos: La técnica de clavo bloqueado ha sido propuesta como un método seguro, con menor tiempo de recuperación, y mejores resultados en el tratamiento de fracturas diafisarias de fémur en perros, por actuar sobre el vector de fuerza del hueso, respetando el mecanismo biológico de reparación de fracturas. Conclusiones: La técnica de clavo bloqueado se muestra aplicable y posible de ejecución para los tratamientos de las fracturas diafisarias de fémur en perros

Palabras-clave: Consolidación de fractura. Fijación de fractura. Fijadores internos. Clavos ortopédicos. Fracturas del fémur. Perros.

\section{Referências}

1. HARASEN, G. Common long bone fractures in small animal practice. Part 1. Canadian Veterinary Journal, v. 44, p. 333-334, 2003.

2. LARIN, A. et al. Repair of diaphyseal femoral fractures in cats using interlocking intramedullary nails: 12 cases (1996-2000). Journal of the American Veterinary Medical Association, v. 219, p. 1098-1104, 2001.

3. PRESNEL, K. R. Pins versus plates: the orthopaedic dilemma. Veterinary Clinics of North America: Small Animal Practice, v. 8, p. 1213-217, 1978.

4. DUHAUTOIS, B. Use of veterinary interlocking nails for diaphyseal fractures in dogs and cats: 121 cases. Veterinary Surgery, v. 32, p. 8-210, 2003.
5. KHAN, F. A. et al. Femoral neck fracture: a complication of femoral nailing. Injury, v. 26, p. 319-321, 1995.

6. ARON, D. N.; PALMER, R. H.; JOHNSON, A. L. Biologic strategies and a balanced concept for repair of highly comminuted long bone fractures. Veterinary Compendium of Orthopedics and Traumatology, v. 7, p. 35-38, 1995.

7. BERNARDÉ, A. et al. An in vitro biomechanical comparison between bone plate and interlocking nail. 3-D interfragmentary motion and bone strain analysis in ostectomized canine femurs. Veterinary Compendium of Orthopedics and Traumatology, v. 15 , p. $57-66.2002$. 
8. PERREN, S. M. The concept of biological plating using the limited contact; dynamic compression plate (LC-DCP): scientific background, design and application. Injury, v. 22, p.1-41, 1991.

9. WOO, S. L. Y.; LOTHRINGER, K. S.; AKESON, W. H. Less rigid internal fixation plates: historical perspectives and new concepts. Journal of Orthopedic Research, v. 1, p. 431-435, 1984.

10. McLAUGHLIN, R. Internal fixation, intramedullary pins, cerclage wires and interlocking nails. Veterinary Clinics of North America: Small Animal Practice, v. 29, p. 1097-1116, 1999.

11. PALMER, R. H. Biological osteosynthesis. Veterinary Clinics of North America: Small Animal Practice, v. 29, p. 1171-1185, 1999.

12. HORSTMAN, C. L.; BEALE, B. S.; CONZEMIUS, M. G. Biological osteosynthesis versus traditional anatomic reconstruction of 20 long-bone fractures using an interlocking nail: 1994-2001. Veterinary Surgery, v. 33, p. 232-237, 2004.

13. McCLURE, S. R.; WATKINS, J. P.; ASHMAN, R. B. In vivo evaluation of intramedullary interlocking nail fixation of transverse femoral osteotomies in foals. Veterinary Surgery, v. 27, p. 29-36, 1998.

14. LOPEZ, M. et al. An in vitro biomechanical comparison of an interlocking nail system and dynamic compression plate fixation of ostectomized equine third metacarpal bones. Veterinary Surgery, v. 28, p. 333-340, 1999.
15. DUELAND, R. T. et al. Interlocking nail treatment of diaphyseal long-bone fractures in dogs. Journal of the American Veterinary Medical Association, v. 214, p. 59-66, 1999.

16. GUPTA, A. Dynamic compression nail: a preliminary report. BMC Musculoskeletal disorders, v. 2, n. 6 , 2001. Disponível em: http://www.biomedcentral.com/ 1471-1474/2/6. Acesso: 01/09/2004.

17. MOSES, P. A. et al. Intramedullary interlocking nail stabilization of 21 humeral fractures in 19 dogs and one cat. Australian Veterinary Journal, v. 80, p. 326-343, 2002.

18. LANGLEY-HOBBS, S. J.; FRIEND, E. Interlocking nail repair of a fractured femur in a turkey. Veterinary Record, v. 150, p. 247-248, 2002.

19. SARAVANAN, B. et al. Management of comminuted femoral fracture by different internal fixation techniques in dogs. Indian Journal of Animal Sciences, v. 72, p. 1104-1107, 2002.

20. SARAVANAN, B. et al. Histopathological evaluation of different internal fixation techniques for the management of comminuted fracture in dog. Indian Journal of Animal Sciences, v. 72, p. 665 $667,2002$.

21. ElShAFIE, M. H.; MORSEY, H. A.; EID, Y. E. Ipsilateral fracture of the femoral neck and shaft, treatment by reconstruction interlocking nail. Archives of Orthopedic and Trauma Surgery, v. 121, p. 71-74, 2001. 